\title{
ON THE NIELSEN-SCHREIER THEOREM IN HOMOTOPY TYPE THEORY
}

\author{
ANDREW W SWAN
}

Department of Philosophy, Carnegie Mellon University, Pittsburgh

e-mail address: andrewsw@andrew.cmu.edu

\begin{abstract}
We give a formulation of the Nielsen-Schreier theorem (subgroups of free groups are free) in homotopy type theory using the presentation of groups as pointed connected 1-truncated types. We show the special case of finite index subgroups holds constructively and the full theorem follows from the axiom of choice. We give an example of a boolean $\infty$-topos where our formulation of the theorem does not hold and show a stronger "untruncated" version of the theorem is provably false in homotopy type theory.
\end{abstract}

\section{INTRODUCTION}

The statement of the Nielsen-Schreier theorem sounds very simple at first: subgroups of free groups are themselves free. However direct proofs are known to be surprisingly intricate and difficult. This was the case for the original proofs by Nielsen [Nie21], for finitely generated free groups, and Schreier [Sch27], generalising to all free groups.

However, later on much clearer proofs were developed based on ideas from topology, the first by Baer and Levi [BL36]. ${ }^{1}$ The idea essentially is that free groups are precisely the fundamental groups of bouquets of circles. Any subgroup is then the fundamental group of a covering space of a bouquet of circles. However, any covering space is homotopic to the geometric realisation of a graph, so the problem is reduced to showing that the fundamental groups of graphs are free groups. This is proved by constructing a spanning tree of the graph, which is then contracted down to point, leaving the remaining edges outside the spanning tree as edges from that point to itself, showing that the graph is homotopy equivalent to a bouquet of circles.

This use of ideas from topology makes the Nielsen-Schreier theorem a natural candidate for formalisation in homotopy type theory [Uni13]. In homotopy type theory we can study spaces from a synthetic point of view, allowing us to use much simpler definitions that are easier to deal with in formalisations, while still being guided by the same topological

Key words and phrases: homotopy type theory, free groups.

I gratefully acknowledge the support of the Air Force Office of Scientific Research through MURI grant FA9550-15-1-0053. Any opinions, findings and conclusions or recommendations expressed in this material are those of the authors and do not necessarily reflect the views of the AFOSR..

${ }^{1}$ There was also a slightly earlier proof by Chevalley and Herbrand [CH31] along similar lines using Riemann surfaces. 
intuitions. We will give a new proof of the Nielsen-Schreier theorem making essential use of types with non trivial higher structure, higher inductive types and univalence, providing an interesting example of a proof using these ideas of a result that is often stated in a concrete purely algebraic way.

Läuchli showed in [Läu62] that the use of some form of the axiom of choice is strictly necessary for the Nielsen-Schreier theorem, by proving that it fails in a Fraenkel-Mostowski model of ZFA. ${ }^{2}$ We will show how this result manifests in homotopy type theory by giving an example of a boolean $\infty$-topos where it is false, together with a stronger "untruncated" version that is provably false in homotopy type theory.

We will assume throughout that the reader is familiar with standard ideas in homotopy type theory such as transport, hlevel, higher inductive types including truncation, connectedness and univalence. See [Uni13] for all of these concepts.

Agda Formalisation. The finite index case (Theorem 5.4) has been verified electronically using the Agda proof assistant and the HoTT-Agda library $\left[\mathrm{BHC}^{+}\right]$. It is available at https://github.com/awswan/nielsenschreier-hott.

Acknowledgements. I am grateful to Mathieu Anel, Steve Awodey, Thierry Coquand and Jonas Frey for helpful discussion and suggestions. I would also like to thank the anonymous referees for their helpful suggestions.

\section{Group Theory and Higher Group Theory in HotT}

In homotopy type theory we can give an alternative definition of group based on the idea of thinking of a group as the fundamental group of some space:

Definition 2.1. A group is a pointed type ( $B G$, base) such that $B G$ is 1-truncated and connected. A group homomorphism $\left(B G\right.$, base $\left._{G}\right) \rightarrow\left(B H\right.$, base $\left._{H}\right)$ is a function $f: B G \rightarrow$ $B H$ together with a proof of $f\left(\right.$ base $\left._{G}\right)=$ base $_{H}$.

One can show that there is an exact correspondence between groups in the above sense, and the more usual definition of group as a set with binary operation satisfying axioms. Given a group ( $B G$, base) as above, we define $G$ to be the loop space $\Omega(B G$, base) := base = base. This has a binary operation - given by composition of paths. As shown by Licata and Finster [LF14], every group in the usual sense is isomorphic, and so by univalence, equal to such a loop space using the higher inductive types of Eilenberg-MacLane spaces.

As shown by Buchholtz, Van Doorn and Rijke [BvDR18], one of the advantages of this approach is that it easily generalises to higher dimensions. In particular we can define $\infty$-groups using a slightly simpler definition:

Definition 2.2. An $\infty$-group is a pointed type ( $B G$, base) such that $B G$ is connected.

We can understand subgroups in this setting using the notion of covering space [HH18].

\footnotetext{
${ }^{2}$ Later on Howard [How85] and Kleppmann [Kle15] gave stronger results that further clarify the precise relationship between the Nielsen-Schreier theorem and the axiom of choice.
} 
Definition 2.3. Let ( $B G$, base) be a group. A covering space on $B G$ is a function $B G \rightarrow$ hSet.

A pointed covering space is a covering space $X: B G \rightarrow \mathbf{h S e t}$ together with an element of $X$ (base).

We say a covering space $X: B G \rightarrow \mathbf{h S e t}$ is connected if the total space $\sum_{z: B G} X z$ is connected.

We say a covering space $X: B G \rightarrow \mathbf{h S e t}$ has index $I$ if there merely exists an equivalence between $X$ (base) and $I$. In particular, we say it has finite index if $X$ (base) is merely equivalent to an initial segment of $\mathbb{N}$.

Pointed connected covering spaces on $B G$ correspond precisely to subgroups of $G$ [BvDR18, Theorem 7.1(3)]. We will therefore sometimes refer to them simply as subgroups.

Free groups in this setting were studied by Kraus and Altenkirch [KA18]. We recall some of their results below.

We first define the free higher group $B F_{A}^{\infty}$ as the higher inductive type generated by the following constructors:

(1) $B F_{A}^{\infty}$ contains a point base.

(2) For each $a: A$ we add a path $\operatorname{loop}(a)$ from base to base.

If $A$ is a set with decidable equality then $B F_{A}$ is 1-truncated, and so a group, as defined above. It is currently an open problem whether this can be proved constructively for sets $A$ in general. We therefore define the free group on $A$ to be the 1-truncation $B F_{A}:=\left\|B F_{A}^{\infty}\right\|_{1}$.

We can equivalently characterise the free group on $A$ using any of the following descriptions.

(1) The 1-truncation of the wedge product of $A$ copies of $\left(\mathbb{S}^{1}\right.$, base).

(2) The coproduct of $A$ copies of the group $\mathbb{Z}:=\left(\mathbb{S}^{1}\right.$, base $)$ in the category of groups and group homomorphisms.

(3) The 1-truncation of the coequalizer of the graph $A \rightrightarrows 1$.

(4) The unique group $\left(B F_{A}\right.$, base) equipped with a map loop : $A \rightarrow \Omega\left(B F_{A}\right.$, base $\left._{F_{A}}\right)$ satisfying the universal property that for any group $\left(B G\right.$, base $\left.{ }_{G}\right)$ and any map $g: A \rightarrow$ $\Omega\left(B G\right.$, base $\left._{G}\right)$, there is a unique homomorphism $h:\left(B F_{A}\right.$, base $\left._{F_{A}}\right) \rightarrow\left(B G\right.$, base $\left._{G}\right)$ such that for all $a: A, \operatorname{ap}_{h}(\operatorname{loop}(a))=g(a)$.

\section{Coequalizers in HoTT}

In this section we review the definition of coequalizers in homotopy type theory and show some useful lemmas. We will omit some of the formal details. See the Agda formalisation for complete proofs. ${ }^{3}$

Definition 3.1. A graph consists of two types $V$ and $E$ together with two maps $\pi_{0}, \pi_{1}$ : $E \rightrightarrows V$. We will refer to elements of $V$ as vertices and elements of $E$ as edges.

Definition 3.2. Suppose we are given a graph $\pi_{0}, \pi_{1}: E \rightrightarrows V$. The coequalizer of $\left(V, E, \pi_{0}, \pi_{1}\right)$, denoted $V / E$ when $\pi_{0}$ and $\pi_{1}$ are clear from the context, is the higher inductive type generated by the following constructors.

(1) For every $v: V, V / E$ contains a point $[v]$.

(2) For every $e: E$ there is a path edge : $\left[\pi_{0}(e)\right]=\left[\pi_{1}(e)\right]$ in $V / E$.

\footnotetext{
${ }^{3}$ These lemmas are in the directory main/Coequalizers.
} 
We will use the following three key lemmas about coequalizers in the proof.

In the first lemma we are given a graph where the type of edges is a coproduct of two types $E_{0}$ and $E_{1}$. We show that the coequalizer $V / E_{0}+E_{1}$ can be computed in two steps, first quotienting by $E_{0}$, and then by $E_{1}$. We can visualise this as follows. Suppose we are given a topological space $V$, and produce a new space by gluing on a set of intervals indexed by $E$. Then we obtain the same space by first gluing on half of the intervals, and then separately gluing on the other half.

Lemma 3.3. Suppose we are given types $E_{0}, E_{1}, V$ together with a pair of maps $\pi_{0}, \pi_{1}$ : $E_{0}+E_{1} \rightrightarrows V$. By composing with the coproduct inclusion we get a diagram $E_{0} \rightrightarrows V$, and so a type $V / E_{0}$ given by coequalizer. We then obtain a pair of maps $E_{1} \rightrightarrows V / E_{0}$ by composing with the other coproduct inclusion and the map $[-]: V \rightarrow V / E_{0}$. We then have the equivalence below.

$$
V /\left(E_{0}+E_{1}\right) \simeq\left(V / E_{0}\right) / E_{1}
$$

Proof. Functions in both directions $f: V /\left(E_{0}+E_{1}\right) \rightarrow\left(V / E_{0}\right) / E_{1}$ and $g:\left(V / E_{0}\right) / E_{1} \rightarrow$ $V /\left(E_{0}+E_{1}\right)$ can be constructed by recursion on coequalizers. One can then show $f \circ g \sim 1$ and $g \circ f \sim 1$ by induction on the construction of the coequalizers.

We visualise the second lemma as follows. We are given a space $X$ together with a point $x \in X$. We extend $X^{\prime}$ to a larger space by adding an extra path $e$ with one endpoint attached at $x$. Then $X^{\prime}$ is homotopy equivalent to $X$, since we can contract the new path $e$ down to the point $x$. The analogous construction in algebraic topology is sometimes known as "growing a whisker."

Lemma 3.4. Let $X$ be a type with an element $x: X$. Define two maps $1 \rightrightarrows X+1$ corresponding to the two elements $\operatorname{inl}(x)$ and $\operatorname{inr}(*)$ of $X+1$. Then the canonical map to the coequalizer $f: X \hookrightarrow X+1 \rightarrow X+1 / 1$ is an equivalence.

Proof. We can construct an inverse $g:(X+1) / 1 \rightarrow X$ by recursion on the definition of coequalizer. Note that $g \circ f$ is definitionally equal to 1 , and we can show $f \circ g \sim 1$ by induction on the definition of $(X+1) / 1$.

Lemma 3.5. "Coequalizers are stable under pullback." Suppose we are given a graph $E \rightrightarrows V$ and a family of types $X: V / E \rightarrow$ Type. Define $E^{\prime}:=\sum_{e: E} X\left(\left[\pi_{0}(e)\right]\right)$ and $V^{\prime}:=$ $\sum_{v: V} X([v])$. Define $\pi_{0}^{\prime}, \pi_{1}^{\prime}: E^{\prime} \rightarrow V^{\prime}$ by $\pi_{0}^{\prime}(e, x):=\left(\pi_{0}(e), x\right)$ and $\pi_{1}^{\prime}(e, x):=\operatorname{edge}(e)_{*}(x)$ Then $\sum_{z: V / E} X(z) \simeq V^{\prime} / E^{\prime}$.

Proof. This can be seen as a special case of the flattening lemma for coequalizers [Uni13, Lemma 6.12.2]. However, for completeness we will give a direct proof.

We first define a map $f: \sum_{z: V / E} X(z) \rightarrow V^{\prime} / E^{\prime}$. Equivalently we can define a dependent function $f^{\prime}: \prod_{z: V / E}\left(X(z) \rightarrow V^{\prime} / E^{\prime}\right)$. We define $f^{\prime}$ using the elimination principle of $V / E$. Given $v: V$, we define $f^{\prime}([v])(x)$ to be $[(v, x)]$. Given $e: E$ we need to define a path as below.

$$
\text { edge }(e)_{*}\left(f^{\prime}\left(\left[\pi_{0}(e)\right]\right)\right)=f^{\prime}\left(\pi_{1}(e)\right)
$$

However, by path induction we can show that for all paths $p: z=z^{\prime}$ in $V / E$ and all $h: X(z) \rightarrow V^{\prime} / E^{\prime}$ that $p_{*}(h)(x)=h\left(p_{*}^{-1}(x)\right)$.

By applying the above with $h=f^{\prime}\left(\pi_{0}(e)\right)$ and $p=$ edge $(e)$, and function extensionality, we can deduce (3.1) by finding for each $e: E$ and each $x: X\left(\pi_{1}(e)\right)$ a path of the type below.

$$
f^{\prime}\left(\left[\pi_{0}(e)\right]\right)\left(\operatorname{edge}(e)_{*}^{-1}(x)\right)=f^{\prime}\left(\left[\pi_{1}(e)\right]\right)(x)
$$


By definition, it suffices to find a path $\left(\pi_{0}(e)\right.$, edge $\left.(e)_{*}^{-1}(x)\right)=\left(\pi_{1}(e), x\right)$ in $V^{\prime}$. However, by the characterisation of identity types for $\sum$-types, this is the same as a path $q: \pi_{0}(e)=\pi_{1}(e)$ together with a path as below.

$$
q_{*}\left(\operatorname{edge}(e)_{*}^{-1}(x)\right)=x
$$

We can of course take $q:=$ edge $(e)$.

We will define $g: V^{\prime} / E^{\prime} \rightarrow \sum_{z: V / E} X(z)$ by recursion on the construction of $V^{\prime} / E^{\prime}$. We define $g([(v, x)]):=([v], x)$. Given $(e, x): E^{\prime}$, we have an evident path $\left(\left[\pi_{0}(e)\right], x\right)=$ $\left(\left[\pi_{1}(e)\right]\right.$, edge $\left.(e)_{*}(x)\right)$, which gives us the well defined function $g$.

Finally, one can verify $f \circ g \sim 1$ by induction on the definition of $V^{\prime} / E^{\prime}$, and $g \circ f \sim 1$ by induction on the definition of $V / E$.

We will often also implicitly use the following lemma.

Lemma 3.6. Suppose we are given graphs $\tau_{0}, \tau_{1}: D \rightrightarrows U$ and $\pi_{0}, \pi_{1}: E \rightrightarrows V$ together with equivalences $D \simeq E$ and $U \simeq V$ commuting with the endpoint maps. Then we have an equivalence $U / D \simeq V / E$.

Proof. For convenience we will assume that all types involved lie at the same universe level. ${ }^{4}$ By univalence we may assume that $D=E$ and $U=V$, and that the equivalences are given by transport along these paths. Hence it suffices to show that for all types $D, E, U, V$, all paths $p: D=E$ and $q: U=V$, all maps $\tau_{0}, \tau_{1}: D \rightrightarrows U$ and $\pi_{0}, \pi_{1}: E \rightrightarrows V$ and finally all proofs that $q_{*} \circ \tau_{i}=\pi_{i} \circ p_{*}$ we have an equivalence $U / D \simeq V / E$. However, the preceding statement can be proved by iterated path induction.

\section{Spanning Trees in HoTT}

We think of the coequalizer of a graph $E \rightrightarrows V$ as its geometric realisation, the topological space that has a point for each vertex $v: V$, and a path from $\pi_{0}(e)$ to $\pi_{1}(e)$ for each edge $e: E$. Note that a graph is connected if and only if its geometric realisation is connected. Similarly a graph is a tree if and only if its geometric realisation is contractible, or equivalently if it is both connected and 0-truncated (contains no non trivial cycles). We will take this topological point of view as the definition of connected and tree.

Definition 4.1. Let $E \rightrightarrows V$ be a graph. We say the graph is connected if $E / V$ is a connected type, and we say the graph is a tree if $E / V$ is contractible.

Definition 4.2. Let $\pi_{0}, \pi_{1}: E \rightrightarrows V$ be a graph. A subgraph is a graph $D \rightrightarrows U$ together with embeddings $h: D \hookrightarrow E$ and $k: U \hookrightarrow V$ such that the following squares commute for $i=0,1$ :

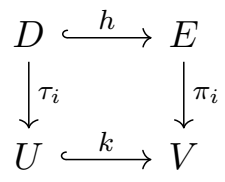

\footnotetext{
${ }^{4}$ For the more general statement where the types can have different universe levels, we need an additional lemma that coequalizers are preserved by "lifting" to higher universe levels. See the file main/Coequalizers/PreserveEquivalence.agda in the formalisation for details.
} 
Definition 4.3. Let $E \rightrightarrows V$ be a graph. A spanning tree is a subgraph $D \rightrightarrows U$ such that $D \rightrightarrows U$ is a tree, the embedding $U \hookrightarrow V$ is an equivalence and the embedding $D \hookrightarrow E$ has decidable image. ${ }^{5}$

We will give two lemmas on the existence of spanning trees. Both will use Lemma 4.5, which in turn uses the lemma below.

Lemma 4.4. Suppose we are given a connected graph $E \rightrightarrows V$, and that $V$ decomposes as a coproduct $V \simeq V_{0}+V_{1}$. Suppose further that we are given an element of each component $v_{0}: V_{0}$ and $v_{1}: V_{1}$. Then there merely exists an edge $e: E$ such that either $\pi_{0}(e) \in V_{0}$ and $\pi_{1}(e) \in V_{1}$, or $\pi_{0}(e) \in V_{1}$ and $\pi_{1}(e) \in V_{0}$.

Proof. We first define a family of propositions $P: V / E \rightarrow$ Type. We wish $P$ to satisfy the following. For $v: V_{0}, P([\operatorname{inl}(v)])=1$, and for $v: V_{1}$ we require the equation below.

$$
P([\operatorname{inr}(v)])=\left\|\sum_{e: E}\left(\pi_{0}(e) \in V_{0} \times \pi_{1}(e) \in V_{1}\right)+\left(\pi_{0}(e) \in V_{1} \times \pi_{1}(e) \in V_{0}\right)\right\|
$$

To show such a $P$ exists, we note that the requirements above precisely define the action on points of a recursive definition on $V / E$. Hence to get a well defined function it suffices to define an action on paths. That is, we need equalities $P\left(\left[\pi_{0}(e)\right]\right)=P\left(\left[\pi_{1}(e)\right]\right)$ for $e: E$. By propositional extensionality we just need to show $P\left(\left[\pi_{0}(e)\right]\right)$ and $P\left(\left[\pi_{1}(e)\right]\right)$ are logically equivalent. However, this is straightforward by considering the 4 cases depending on whether $\pi_{i}(e) \in V_{0}$ or $\pi_{i}(e) \in V_{1}$ for $i=0,1$ : if $\pi_{0}(e)$ and $\pi_{1}(e)$ lie in the same component of the coproduct, then $P\left(\left[\pi_{0}(e)\right]\right)$ and $P\left(\left[\pi_{1}(e)\right]\right)$ are the same by definition, and if they lie in different components, then $P([v])$ is true for all $v: V$. We now construct a map from $\left[\operatorname{inl}\left(v_{0}\right)\right]=z$ to $P(z)$ for each $z: V / E$. By based path induction it suffices to construct an element of $P\left(\operatorname{inl}\left(v_{0}\right)\right)$, but this was defined to be 1 , so is trivial. By connectedness, there merely exists an element of $\left[\operatorname{inl}\left(v_{0}\right)\right]=\left[\operatorname{inl}\left(v_{1}\right)\right]$, and so $P\left(\operatorname{inl}\left(v_{1}\right)\right)$ is inhabited and the lemma follows.

To further illustrate the proof of Lemma 4.4 we give an alternative non constructive proof using the same family of types $P$.

Using the law of excluded we can assume there is no edge with endpoints in different components of $V$ and derive a contradiction. Under this assumption, our requirement on $P$ is that it is 1 on $V_{0}$ and 0 on $V_{1}$. In other words we have a 2-colouring of vertices and want to extend it to a 2-colouring on the whole graph. We can do this by the assumption, since any edge has the same colour on both its endpoints, so we can take the whole edge to be that colour. This now contradicts connectedness, since we have a surjection from the graph to 2. Topologically, we can think of this as a continuous surjection from a connected space to the discrete space 2 , which is not possible.

Lemma 4.5. Let $E \rightrightarrows V$ be a connected graph where $V$ has decidable equality and $E$ is a set, together with a subgraph $D \rightrightarrows U$ such that the inclusion $U \hookrightarrow V$ has decidable image.

Suppose further that we are given elements $u \in U$ and $v \in V \backslash U$. Then there merely exists a larger subgraph whose type of vertices is $U+1$ and whose type of edges is $D+1$,

\footnotetext{
${ }^{5}$ It is not clear if decidable image should be part of the definition of spanning tree for every application. However, the main use of spanning trees for this paper will be Lemma 4.8 below, where it does play a critical role.
} 
such that the canonical map $U / D \rightarrow(U+1) /(D+1)$ is an equivalence, as illustrated below:

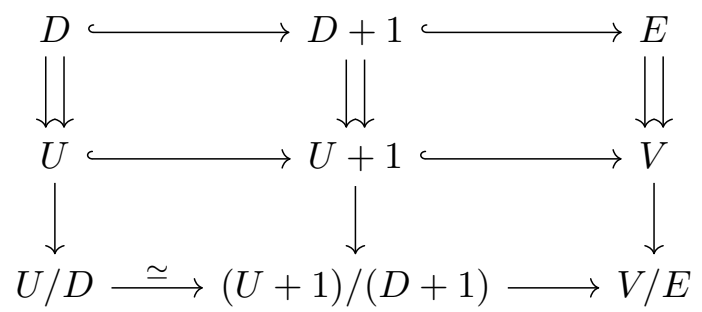

Proof. Since the inclusion $U \hookrightarrow V$ is decidable, we can write $V$ as the coproduct of $U$ with its complement $V \simeq U+V \backslash U$. Applying Lemma 4.4 shows there merely exists an edge $e: E$ such that either $\pi_{0}(e) \in U$ and $\pi_{1}(e) \notin U$ or vice versa. We consider the former case, the latter being similar.

We define the map $U+1 \hookrightarrow V$ to be the same as the map $U \hookrightarrow V$ on the $U$ component and to be equal to $\pi_{1}(e)$ on the 1 component. Since $\pi_{1}(e) \notin U$, this is an embedding. Similarly we define the map $D+1 \hookrightarrow E$ by taking the 1 component to $e$. Note that we cannot have $e \in D$, since this would imply $\pi_{1}(e) \in U$, and so the map $D+1 \hookrightarrow E$ is also an embedding.

We define the endpoint maps $D+1 \rightrightarrows U+1$ as appropriate to satisfy commutativity conditions.

Finally, we verify the equivalence by computing as follows.

$$
\begin{aligned}
(U+1) /(D+1) & \simeq((U+1) / 1) / D & & \text { Lemma } 3.3 \\
& \simeq U / D & & \text { Lemma } 3.4
\end{aligned}
$$

Lemma 4.6. Let $E \rightrightarrows V$ be a connected graph where $V$ is finite, say with $|V|=n$ and $E$ is a set with decidable equality. Then the graph merely has a spanning tree $D \hookrightarrow E \rightrightarrows V$ where $D$ is finite with $|D|=n-1$.

Proof. We show by induction that for $1 \leq k \leq n$ there merely exists a subgraph $D \rightrightarrows U$ such that $|U|=k,|D|=k-1$ and $U / D$ is contractible.

For $k=1$ we observe that by the definition of connectedness, $V / E$ is merely inhabited, and so $V$ is also merely inhabited. An element $v$ of $V$ defines an embedding $1 \hookrightarrow V$, and it is clear that the coequalizer $1 / 0$ is contractible.

Now suppose we have already defined a suitable subgraph for $1 \leq k<n$, say $D \rightrightarrows U$. Since $V$ is finite, it in particular has decidable equality. Furthermore, since $|U|=k$ with $1 \leq k<n$, there exist $u \in U$ and $v \notin U$. Hence we can apply Lemma 4.5 to show the existence of a subgraph of the form $D+1 \rightrightarrows U+1$ where $(U+1) /(D+1) \simeq U / D$. Since $U / D$ is contractible, so is $(U+1) /(D+1)$, as required.

Now we apply the above with $k=n$ to get a subgraph $D \rightrightarrows U$ where $U / D$ is contractible. Since $U$ and $V$ are both finite of the same size $n$, the embedding $U \hookrightarrow V$ is an equivalence. Since $E$ has decidable equality and $D$ is finite, the embedding $D \hookrightarrow E$ has decidable image. Hence this does indeed give a subtree.

Lemma 4.7. Let $E \rightrightarrows V$ be a connected graph where $E$ and $V$ are both sets. Suppose that the axiom of choice holds. Then a spanning tree for the graph merely exists.

Proof. Recall that the axiom of choice implies the law of excluded middle and Zorn's lemma. We consider the set of subgraphs of $E \rightrightarrows V$ that are trees, ordered by inclusion. 
We verify that the poset is chain complete. If we are given a chain of subgraphs $\left(D_{i} \rightrightarrows U_{i}\right)_{(i: I)}$, take $D \rightrightarrows U$ to be the union of all the subgraphs, and write $\iota_{i}$ for the canonical map $U_{i} / D_{i} \rightarrow U / D$. Fix $u: U$, noting that such a $u$ merely exists since $I$ is merely inhabited ${ }^{6}$ and $U_{i} / D_{i}$ is contractible for each $i$. For $v: U$, we choose ${ }^{7} i: I$ such that $u, v \in U_{i}$, and take $p$ to be the unique path $[u]=[v]$ in $U_{i}$. This then gives us a path $\iota_{i}(p)$ in $U / D$. Similarly, for each $e: D$, we can choose an element $i: I$ such that $e \in D_{i}$, and from this construct a homotopy between the choice of path from $[u]$ to $\left[\pi_{0}(e)\right]$ composed with $e$ and the choice of path from $[u]$ to $\left[\pi_{1}(e)\right]$. Combined with the induction principle for $U / D$, this gives us a path from $[u]$ to $z$ for each $z: U / D$, showing that $U / D$ is contractible.

Hence the poset has a maximal element $D \rightrightarrows U$ by Zorn's lemma. We wish to show every element of $V$ belongs to $U$. By the law of excluded middle, it suffices to derive a contradiction from the assumption of $v \in V \backslash U$. However, by Lemma 4.5 we could obtain a larger tree subgraph, contradicting maximality, as required.

Again using excluded middle, $D$ has a complement in $E$, giving us the spanning tree.

We next see a key lemma that establishes the geometric realisation of graphs that have spanning trees are equivalent to bouquets of circles. The way to visualise this is that we contract the spanning tree down to a single point. This leaves the remaining edges not in the spanning tree as loops from this single point to itself.

Lemma 4.8. Suppose that $E \rightrightarrows V$ is a graph with a spanning tree $E_{0} \hookrightarrow E_{0}+E_{1} \simeq E$. Then $V /\left(E_{0}+E_{1}\right)$ is equivalent to the free $\infty$-group on $E_{1}$.

Proof.

$$
\begin{aligned}
V /\left(E_{0}+E_{1}\right) & \simeq\left(V / E_{0}\right) / E_{1} \\
& \simeq 1 / E_{1} \\
& \simeq B F_{E_{1}}^{\infty}
\end{aligned}
$$

Lemma 3.3

since $E_{0}$ is a spanning tree

\section{The Nielsen-Schreier Theorem}

We now prove two versions of the Nielsen-Schreier theorem in HoTT. Following the classical proofs, we proceed in two steps. We first show that every subgroup of a free group is equivalent to the geometric realisation of a graph. We then use the results of Section 4 to deduce that it equivalent to a free group, under certain conditions.

Lemma 5.1. "Every bundle on a free $\infty$-group is the geometric realisation of a graph." Let $A$ be any type, and $\left(B F_{A}\right.$, base) the free $\infty$-group on $A$ generated by paths loop $(a)$ for $a: A$. Let $X: B F_{A} \rightarrow$ Type be any family of types over $B F_{A}$. We define a graph $\pi_{0}, \pi_{1}: A \times X$ (base) $\rightarrow X$ (base) by taking $\pi_{0}$ to be projection, and define $\pi_{1}(a, x)$ to be $\operatorname{loop}(a)_{*}(x)$. We then have

$$
\sum_{z: B F_{A}^{\infty}} X(z) \simeq X(\text { base }) /(A \times X(\text { base }))
$$

\footnotetext{
${ }^{6}$ We follow the convention that chains are inhabited.

${ }^{7}$ This requires an application of the axiom of choice to inhabited subsets of $I$.
} 
Proof. Note that $B F_{A}$ is equivalent to the coequalizer of a graph $A \rightrightarrows 1$ with base $=[*]$ where $*$ is the unique element of 1 . Hence we can apply Lemma 3.5 to express $\sum_{z: B F_{A}} X(z)$ as a coequalizer $V^{\prime} / E^{\prime}$. However, we then have the following definitional equalities and equivalences.

$$
\begin{gathered}
V^{\prime} \equiv \sum_{v: 1} X([*]) \simeq X([*]) \equiv X(\text { base }) \\
E^{\prime} \equiv \sum_{a: A} X\left(\left[\pi_{0}(1)\right]\right) \equiv \sum_{a: A} X([*]) \equiv \sum_{a: A} X(\text { base }) \simeq A \times X(\text { base })
\end{gathered}
$$

In order to derive the truncated version of Lemma 5.1 we first recall the following flattening lemma for truncation.

Lemma 5.2. Suppose we are given a type $Y$ and a family of sets $X:\|Y\|_{1} \rightarrow \mathbf{h S e t}$. Then $\sum_{z:\|Y\|_{1}} X(z) \simeq\left\|\sum_{y: Y} X\left(|y|_{1}\right)\right\|_{1}$.

Proof. Similar to the proof of Lemma 3.5.

Lemma 5.3. "Every subgroup of a free group is the geometric realisation of a graph." Let $A$ be a set, and $\left(B F_{A}\right.$, base $)$ the free group on $A$. Let $X: B F_{A} \rightarrow \mathbf{h S e t}$ be a covering space on $B F_{A}$. Then we then have the following.

$$
\sum_{z: B F_{A}} X(z) \simeq \| X(\text { base }) /(A \times X(\text { base })) \|_{1}
$$

Proof. We define $X^{\prime}: B F_{A}^{\infty} \rightarrow$ Type to be the composition of $X$ with the truncation map $|-|_{1}: B F_{A}^{\infty} \rightarrow B F_{A}$ and projection from hSet to Type. Lemma 5.1 then gives us an equivalence $\sum_{z: B F_{A}^{\infty}} X^{\prime}(z) \simeq X^{\prime}$ (base $) /\left(A \times X^{\prime}\right.$ (base)). However, $X^{\prime}$ (base $) /(A \times$ $X^{\prime}$ (base $\left.)\right)$ is definitionally equal to $X$ (base $) /(A \times X$ (base) $)$. By Lemma 5.2 we have that $\sum_{z:\left\|B F_{A}^{\infty}\right\|_{1}} X(z)$ is equivalent to $\left\|\sum_{z: B F_{A}^{\infty}} X^{\prime}(z)\right\|_{1}$. Putting these together gives us the required equivalence $\sum_{z:\left\|B F_{A}^{\infty}\right\|_{1}} X(z) \simeq \| X($ base $) /(A \times X($ base $)) \|_{1}$.

We now give two versions of the Nielsen-Schreier theorem that hold in homotopy type theory. The first is entirely constructive and includes the Nielsen-Schreier index formula.

Theorem 5.4. Suppose that $B F_{A}$ is the free group on a set $A$ with decidable equality. Suppose that $X: B F_{A} \rightarrow \mathbf{h S e t}$ is a finite index, connected covering space. Then $\sum_{z: B F_{A}} X z$ is merely equivalent to the classifying space of a free group.

Moreover, suppose that $A$ is finite of size $n$ and that $X$ is of finite index $m$. Then there merely exists an equivalence,

$$
\sum_{z: B F_{A}} X z \simeq B F_{m(n-1)+1}
$$

Proof. By Lemma 5.3 we have $\sum_{z: B F_{A}} X(z) \simeq \| X($ base $) /(A \times X($ base $)) \|_{1} \cdot{ }^{8}$ Note that $X$ (base) $/(A \times X$ (base $))$ is a coequalizer where the vertex set $X$ (base) is finite and the edge set $A \times X$ (base) has decidable equality. Hence we can apply Lemma 4.6 to show a spanning tree exists. Hence we can apply Lemma 4.8 to show $X$ (base $) /(A \times X($ base $))$ is equivalent to a free higher group. Truncating gives us an equivalence between $X($ base $) /(A \times X($ base $))$ and a free group.

\footnotetext{
${ }^{8}$ In fact, since $A$ has decidable equality $X$ (base $) /(A \times X$ (base $\left.)\right)$ is already 1 -truncated, but we will not need that here.
} 
Now suppose that $A$ is also finite, with $|A|=n$ and $\mid X$ (base) $\mid=m$. Then Lemma 4.6 in fact gives us a spanning tree with $m$ vertices and $m-1$ edges. In particular we can write the edge set $A \times X$ (base) as a coproduct $E_{0}+E_{1}$ where $X$ (base)/E $E_{0}$ is contractible and $\left|E_{0}\right|=m-1$. Lemma 4.8 then tells us $X$ (base) $/(A \times X$ (base) $)$ is equivalent to the free (higher) group on $E_{1}$. However $\left|E_{1}\right|=m n-(m-1)=m(n-1)+1$, as required.

Theorem 5.5. Assume the axiom of choice. Suppose that $B F_{A}$ is the free group on a set $A$. Let $X: B F_{A} \rightarrow$ hSet be any connected covering space. Then $\sum_{z: B F_{A}} X z$ is merely equivalent to a free group.

Proof. Similar to the proof of Theorem 5.4 using Lemmas 5.3, 4.7 and 4.8.

\section{A Boolean $\infty$-Topos Where the Theorem does not Hold}

We recall that Läuchli proved the following theorem in [Läu62, Section IV].

Theorem 6.1 (Läuchli). The Nielsen-Schreier theorem is not provable in ZFA, ZermeloFraenkel set theory with atoms.

We will sketch out how to adapt the proof to obtain a model of homotopy type theory with excluded middle where the Nielsen-Schreier theorem does not hold. In order to do this we will work in a classical metatheory and we will switch back to the classical definition of free groups using reduced words. In the presence of the law of excluded middle this is equivalent to our earlier definition by [KA18, Section 2.2].

We first recall the following lemma from Läuchli's proof.

Lemma 6.2. Let $F_{A}$ be a free group and $C \leq F_{A}$ the subgroup generated by elements of the form $a b a^{-1} b^{-1}$ for $a, b \in A$. Let $X \subseteq C$ be a set that freely generates $C$. Then $X$ cannot be invariant under any transposition $(a b)$ for $a \neq b \in A$.

Proof. See [Läu62, Section IV].

We will construct our example using the (1-)topos of nominal sets [Pit13]. We first recall the basic definitions. We fix a countably infinite set $\mathbb{A}$. We write $\operatorname{Perm}(\mathbb{A})$ for the group of finitely supported permutations of $\mathbb{A}$ (i.e. $\pi: A \stackrel{\sim}{\longrightarrow} A$ such that $\pi(a)=a$ for all but finitely many $a \in \mathbb{A})$.

If $(X, \cdot)$ is a $\operatorname{Perm}(\mathbb{A})$-set, $x \in X$ and $A \subseteq \mathbb{A}$, we say $A$ is a support for $x$ if $\pi \cdot x=x$ whenever $\pi \in \operatorname{Perm}(\mathbb{A})$ satisfies $\pi(a)=a$ for all $a \in A$.

The topos of nominal sets is defined to be the full subcategory of $\operatorname{Perm}(\mathbb{A})$-sets consisting of $(X, \cdot)$ such that every element $x \in X$ has a finite support.

Theorem 6.3. The Nielsen-Schreier theorem is false in the internal logic of the topos of nominal sets.

Proof. First recall that $\mathbb{A}$ can itself be viewed as a nominal set by taking the action to be $\pi \cdot a:=\pi(a)$. Take $F_{\mathbb{A}}$ to be the internal free group on $\mathbb{A}$. Observe, e.g. by verifying the universal property that $F_{\mathbb{A}}$ is just the external definition of $F_{\mathbb{A}}$ together with the action given by the action on $\mathbb{A}$ and the universal property.

We define $C \leq F_{\mathbb{A}}$ by externally taking it to be the subgroup generated by elements of the form $a b a^{-1} b^{-1}$ for $a, b \in \mathbb{A}$. We observe that the action of $\operatorname{Perm}(\mathbb{A})$ on $F_{\mathbb{A}}$ restricts to $C$, giving us a subgroup $C \leq F_{\mathbb{A}}$ in nominal sets. The object of freely generating subsets, 
$\mathcal{G}$ of $C$ can be explicitly described as the set of $X \subseteq C$ such that $X$ has finite support and (externally) freely generates $C$, with the obvious action. The Nielsen-Schreier theorem implies that $\mathcal{G}$ contains some element $X$. Let $A \subseteq \mathbb{A}$ be a finite support for $X$. Let $a, b$ be distinct elements of $\mathbb{A} \backslash A$. Then $(a b) \cdot X=X$, contradicting Lemma 6.2.

Corollary 6.4. There is a boolean $\infty$-topos where the Nielsen-Schreier theorem does not hold.

The Nielsen-Schreier theorem is not provable in homotopy type theory, even with the addition of the law of excluded middle.

Proof. The topos of nominal sets is equivalent to a Grothendieck topos referred to as the Schanuel topos [Pit13, Section 6.3].

Lurie showed in [Lur09, Proposition 6.4.5.7] that any Grothendieck (1-)topos is equivalent to the 0 -truncated elements of some Grothendieck $\infty$-topos. In particular, if we apply this to the topos of nominal sets we obtain a boolean Grothendieck $\infty$-topos where Nielsen-Schreier does not hold.

Shulman proved in [Shu19] that homotopy type theory can be interpreted in any Grothendieck $\infty$-topos. We thereby obtain a model of HoTT with the law of excluded middle where Nielsen-Schreier does not hold.

Remark 6.5. As an alternative to the non constructive methods of Lurie and Shulman, it may also be possible to use a cubical sheaf model, as developed by Coquand, Ruch and Sattler [CRS19], but we leave a proof for future work.

\section{The Untruncated Nielsen-Schreier Theorem is False}

In this section we will again work with the more usual definition of group as set with a binary operation satisfying the well known axioms. We will write the free group as $F_{A}$, which is the loop space of $B F_{A}$ at base. We recall the observation of Kraus and Altenkirch [KA18, Section 2.2] that when $A$ has decidable equality we can use the classical description of $F_{A}$ as the set of reduced words with multiplication given by concatenation followed by reduction. In particular we note that when $A$ has decidable equality so does $F_{A}$.

In Theorems 5.4 and 5.5 we were careful to state that the equivalences merely exist, to emphasise that formally we are only constructing an element of the truncation $\left\|\sum_{z: B F_{A}} X z \simeq B F_{B}\right\|$, not the type $\sum_{z: B F_{A}} X z \simeq B F_{B}$ itself. In this section we will see why the distinction is important. To understand this, we first define the following two variants of the Nielsen-Schreier theorem.

Definition 7.1. We say the untruncated Nielsen-Schreier theorem holds if for each set $A$ and each subgroup $H \hookrightarrow F_{A}$ we can choose a subset $C_{A, H} \hookrightarrow H$ that freely generates $H$, i.e. the lift $F_{C_{A, H}} \rightarrow H$ given by universal property is an isomorphism.

Definition 7.2. We say the equivariant Nielsen-Schreier theorem holds if for each set $A$ and each subgroup $H \hookrightarrow F_{A}$ we can choose a subset $C_{A, H} \hookrightarrow H$ that freely generates $H$ satisfying the following condition. Given any equivalence $\pi: A \rightarrow A^{\prime}$ we write $\tilde{\pi}$ for the lift to an isomorphism $F_{A} \cong F_{A^{\prime}}$ given by universal property. We require that $C_{A^{\prime}, \tilde{\pi}(H)}=\tilde{\pi}\left(C_{A, H}\right)$.

Lemma 7.3. The untruncated Nielsen-Schreier theorem implies the equivariant NielsenSchreier theorem.

Moreover, the untruncated Nielsen-Schreier theorem restricted to free groups generated by merely finite sets implies the equivariant Nielsen-Schreier theorem with the same restriction. 
Proof. Given sets $A, A^{\prime}$ and a path $p: A=A^{\prime}$, write idtoeqv $(p)$ for the equivalence $A \rightarrow A^{\prime}$ given by transport. It is straightforward to show by path induction that $C_{A^{\prime}, \text { idtoeqv }(p)(H)}=$ idtoeqv $(p)\left(C_{A, H}\right)$. However, by univalence and the fact that the projection from hSet to Type is an embedding, for every equivalence $\pi: A \simeq A^{\prime}$, there is a unique path $p: A=A^{\prime}$ such that $\pi=$ idtoeqv $(p)$, so this is true for all equivalences.

Note that the projection from merely finite sets to sets is an embedding, so it remains true for merely finite $A, A^{\prime}$ that any $\pi: A \simeq A^{\prime}$ can be written uniquely as idtoeqv $(p)$ for some $p: A=A^{\prime}$.

At this point it is possible to apply Lemma 6.2 to show the equivariant Nielsen-Schreier theorem is false. However, for the theorem below we will use an alternative proof, for two reasons. Firstly, we will give a stronger result that the equivariant version of the theorem fails already for the case of finite index subgroups of finitely generated free groups, whereas in Läuchli's example the free group is infinitely generated, and we can see by Theorem 5.4 the given subgroup must have infinite index. Secondly, we can use an easier, more straightforward argument, since to find a counterexample to equivariance, we only need to show any set of generators is not fixed by some transposition, rather than the stronger result that any generating set is not fixed by any non-trivial transposition. In fact we will see that one of the very simplest instances of the Nielsen-Schreier theorem suffices to find a counterexample.

Theorem 7.4. The equivariant Nielsen-Schreier theorem is false in homotopy type theory, extensional type theory and classical mathematics. Moreover, it remains false if we restrict to finite index subgroups of finitely generated free groups.

Proof. We will given an example of a finite index subgroup of a finitely generated free group $F_{A}$ that does not satisfy the equivariance condition for all automorphisms $\pi: A \simeq A$.

We take $A$ to be a type with exactly two elements that we write as $a$ and $b$. Note that we have a unique homomorphism $\theta: F_{A} \rightarrow \mathbb{Z} / 2 \mathbb{Z}$ that sends both $a$ and $b$ to 1 . We take $H \leq F_{A}$ to be the kernel of $\theta$. We first observe that $H$ has index 2, since for homomorphisms $\theta$ in general, the index of $\operatorname{ker}(\theta)$ is equal to the size of the image of $\theta$, and in this case $\theta$ is surjective and the codomain has size 2 .

Take $\pi$ to be the transposition $(a b)$. By the assumption of equivariant Nielsen-Schreier we have $C_{A, H}$ such that $\tilde{\pi}\left(C_{A, H}\right)=C_{A, \tilde{\pi}(H)}$. However, note that $\theta(\tilde{\pi}(g))=\theta(g)$ for all $g \in F_{A}$, since this is clearly true whenever $g \in A$. Hence $\tilde{\pi}(H)=H$. It follows that $\tilde{\pi}\left(C_{A, H}\right)=C_{A, H}$.

We next show that $C_{A, H}$ has exactly 3 elements. Certainly $H$ can be freely generated by a (non-equivariant) set of 3 elements by the Nielsen-Schreier theorem, say $g_{1}, g_{2}, g_{3} \cdot{ }^{9}$ We only need finitely many elements of $C_{A, H}$ to generate each of $g_{1}, g_{2}, g_{3}$, and so a finitely enumerable subset ${ }^{10}$ of $C_{A, H}$ generates all of $H$. By freeness and the fact that $F_{A}$ has decidable equality, it follows $C_{A, H}$ itself is finitely enumerable. Again using that $F_{A}$ has decidable equality it follows that $C_{A, H}$ is finite, i.e. in bijection with an initial segment of $\mathbb{N}$. One can then show $C_{A, H}$ has the same size as any other finite set that freely generates $H$ using standard arguments from algebra such as [Rot03, Proposition 5.75], which is constructively valid as stated. Hence $\left|C_{A, H}\right|=3$.

We can now deduce that $\tilde{\pi}$ permutes the set with three elements $C_{A, H}$. However, it has order 2 , and any permutation of 3 elements of order 2 has a fixed point. We deduce that

\footnotetext{
${ }^{9}$ In fact we can read off from the proof an explicit set $\left\{a^{2}, a b, a b^{-1}\right\}$.

${ }^{10}$ That is, the surjective image of an initial segment of $\mathbb{N}$.
} 
$\tilde{\pi}(h)=h$ for some $h \in C_{A, H}$. But, using the explicit description of $F_{A}$ in terms of reduced words, the only element of $F_{A}$ fixed by $\tilde{\pi}$ is the identity, which cannot appear in any free generating set, giving a contradiction.

Corollary 7.5. The untruncated Nielsen-Schreier theorem is false in homotopy type theory. Moreover, it remains false if we restrict to finite index subgroups of finitely generated free groups.

Proof. By Lemma 7.3 it suffices to show the equivariant Nielsen-Schreier theorem in finite index subgroups of finitely generated free groups is false, which was Theorem 7.4.

\section{Conclusion}

We have given a proof of two versions of the Nielsen-Schreier theorem in homotopy type theory. The proof of the finite index version, Theorem 5.4 has been verified electronically in the Agda proof assistant using the HoTT Agda library $\left[\mathrm{BHC}^{+}\right]$. Since we only used axioms that are always present in homotopy type theory, the formal proof holds in a wide variety of models. Alternatively, we could have used the new cubical mode now available in Agda [VMA19]. In this case the class of models is currently limited to those following the Orton-Pitts approach [OP16], but some of the proofs would have been a bit easier (see below).

In the proof we made good use of concepts in HoTT to transfer ideas from the topological proof into type theory. In particular we used the coequalizer higher inductive type to represent the geometric realisation of graphs and (in particular) free groups. This approach was even useful in the relatively concrete construction of spanning trees in Section 4. We defined trees and connected graphs by applying the existing concepts of contractibility and connectedness of types to the coequalizer of the graph. We were able to work directly with these definitions throughout, without ever needing to define or use the more usual notion of path in a graph as a finite sequence of edges, which was an advantage in the electronic formalisation.

One minor difficulty in the formal proof is the lemmas on coequalizers appearing in Section 3, in particular the higher paths that appear when constructing the equality part of an equivalence by coequalizer induction. However, many of these difficulties could have been eliminated by instead using cubical mode. This allows one to define coequalizers using the usual Agda data syntax, to use pattern matching instead of elimination terms and it makes $\beta$-reduction for path constructors definitional, the latter playing a useful role in these proofs particularly. To demonstrate this a formalisation of some of the results of Section 3 using cubical mode has been added in a separate directory.

The independence result in Section 6 shows that the axiom of choice is necessary for the main theorem. It also demonstrates that independence results in HoTT can now be relatively straightforward thanks to Shulman's interpretation of HoTT in a Grothendieck $\infty$-topos [Shu19], Lurie's construction of enveloping $\infty$-toposes [Lur09] and the existing body of work on Grothendieck toposes, in this case the use of nominal sets to provide simpler categorical versions of proofs using Fraenkel-Mostowski models.

Finally the result in Section 7 provides a simple example of an important concept in HoTT and its relevance to the Nielsen-Schreier theorem: mathematics in the presence of univalence is "inherently equivariant." 


\section{REFERENCES}

$\left[\mathrm{BHC}^{+}\right] \quad$ Guillaume Brunerie, Kuen-Bang Hou (Favonia), Evan Cavallo, Tim Baumann, Eric Finster, Jesper Cockx, Christian Sattler, Chris Jeris, Michael Shulman, et al. Homotopy type theory in Agda. URL: https://github. com/HoTT/HoTT-Agda.

[BL36] Reinhold Baer and Friedrich Levi. Freie Produkte und ihre Untergruppen. Compositio Mathematica, 3:391-398, 1936. URL: http://www.numdam.org/item/CM_1936__3__391_0.

[BvDR18] Ulrik Buchholtz, Floris van Doorn, and Egbert Rijke. Higher groups in homotopy type theory. In Proceedings of the 33rd Annual ACM/IEEE Symposium on Logic in Computer Science, LICS '18, page 205-214, New York, NY, USA, 2018. Association for Computing Machinery. doi: 10.1145/3209108.3209150.

[CH31] Claude Chevalley and J. Herbrand. Groupes topologiques, groupes fuchsiens, groupes libres. CR Acad. Sci. Paris, 192:724-726, 1931.

[CRS19] Thierry Coquand, Fabian Ruch, and Christian Sattler. Constructive sheaf models of type theory. arXiv:1912.10407, December 2019.

[HH18] Kuen-Bang Hou (Favonia) and Robert Harper. Covering Spaces in Homotopy Type Theory. In Silvia Ghilezan, Herman Geuvers, and Jelena Ivetić, editors, 22nd International Conference on Types for Proofs and Programs (TYPES 2016), volume 97 of Leibniz International Proceedings in Informatics (LIPIcs), pages 11:1-11:16, Dagstuhl, Germany, 2018. Schloss Dagstuhl-LeibnizZentrum fuer Informatik. URL: http://drops.dagstuhl.de/opus/volltexte/2018/9851, doi: 10.4230/LIPICS. TYPES.2016.11.

[Hig05] Peter J. Higgins. Categories and Groupoids. Number 7 in Reprints in Theory and Applications of Categories. Theory and Applications of Categories, 2005. Originally published by Van Nostrand Reinhold, 1971.

[How85] Paul E. Howard. Subgroups of a free group and the axiom of choice. The Journal of Symbolic Logic, 50(2):458-467, 1985. URL: http://www.jstor.org/stable/2274234.

[KA18] Nicolai Kraus and Thorsten Altenkirch. Free higher groups in homotopy type theory. In Proceedings of the 33rd Annual ACM/IEEE Symposium on Logic in Computer Science, LICS '18, page 599-608, New York, NY, USA, 2018. Association for Computing Machinery. doi:10.1145/3209108. 3209183.

[Kle15] Philipp Kleppmann. Nielsen-Schreier and the axiom of choice. Mathematical logic quarterly, 61(6):458-465, 2015.

[Läu62] H. Läuchli. Auswahlaxiom in der Algebra. Commentarii Mathematici Helvetici, 37:1-18, 1962. doi:10.1007/BF02566957.

[LF14] Daniel R. Licata and Eric Finster. Eilenberg-MacLane spaces in homotopy type theory. In Proceedings of the Joint Meeting of the Twenty-Third EACSL Annual Conference on Computer Science Logic (CSL) and the Twenty-Ninth Annual ACM/IEEE Symposium on Logic in Computer Science (LICS), CSL-LICS '14, New York, NY, USA, 2014. Association for Computing Machinery. doi:10.1145/2603088. 2603153.

[Lur09] Jacob Lurie. Higher Topos Theory. Number 170 in Annals of Mathematics Studies. Princeton University Press, 2009.

[Nie21] J. Nielsen. Om regning med ikke-kommutative faktorer og dens anvendelse i gruppeteorien. (Über das Rechnen mit nicht-vertauschbaren Faktoren und dessen Anwendung in der Gruppentheorie.). Mat. Tidsskr. B, 1921:78-94, 1921.

[OP16] I. Orton and A. M. Pitts. Axioms for modelling cubical type theory in a topos. In J.-M. Talbot and L. Regnier, editors, 25th EACSL Annual Conference on Computer Science Logic (CSL 2016), volume 62 of Leibniz International Proceedings in Informatics (LIPIcs), pages 24:1-24:19, Dagstuhl, Germany, 2016. Schloss Dagstuhl-Leibniz-Zentrum für Informatik. doi:http://dx.doi.org/10. 4230/LIPIcs . CSL . 2016. 24.

[Pit13] A. M. Pitts. Nominal Sets: Names and Symmetry in Computer Science, volume 57 of Cambridge Tracts in Theoretical Computer Science. Cambridge University Press, 2013.

[Rot03] Joseph J. Rotman. Advanced Modern Algebra. Prentice Hall, 2003.

[Sch27] Otto Schreier. Die Untergruppen der freien Gruppen. Abhandlungen aus dem Mathematischen Seminar der Universität Hamburg, 5:161-183, 1927. doi:10.1007/BF02952517.

[Shu19] Michael Shulman. All ( $\infty, 1)$-toposes have strict univalent universes. arXiv:1904.07004, April 2019. 
[Uni13] Univalent Foundations Program. Homotopy Type Theory: Univalent Foundations of Mathematics. http://homotopytypetheory.org/book, Institute for Advanced Study, 2013.

[VMA19] Andrea Vezzosi, Anders Mörtberg, and Andreas Abel. Cubical agda: A dependently typed programming language with univalence and higher inductive types. Proc. ACM Program. Lang., 3(ICFP), July 2019. doi:10.1145/3341691. 